\title{
Structural and electrical characterisation of ion-implanted strained silicon
}

\author{
K. Horan ${ }^{\mathrm{a}, *}$, A. Lankinen ${ }^{\mathrm{b}}$, L. O'Reilly ${ }^{\mathrm{a}}$, N.S. Bennett ${ }^{\mathrm{c}, \mathrm{d}}$, P.J. McNally ${ }^{\mathrm{a}}$, \\ B.J. Sealy ${ }^{\mathrm{d}}$, N.E.B. Cowern ${ }^{\mathrm{c}}$, T.O. Tuomi ${ }^{\mathrm{b}}$ \\ a Nanomaterials Processing Laboratory, Research Institute for Networks and Communications Engineering (RINCE), \\ School of Electronic Engineering, Dublin City University, Dublin 9, Ireland \\ ${ }^{\mathrm{b}}$ Micro and Nanosciences, Micronova, Helsinki University of Technology, P.O. Box 3500, FIN-02015 TKK, Finland \\ ' School of Electronic, Electrical and Computer Engineering, University of Newcastle, Newcastle upon Tyne NE1 7RU, UK \\ d Advanced Technology Institute, University of Surrey, Guildford GU2 7XH, UK
}

\section{A R T I C L E I N F O}

\section{Article history:}

Received 5 May 2008

Received in revised form 19 August 2008

Accepted 1 September 2008

\section{Keywords:}

Micro-Raman

SXRT

HR-XRD

Tensile strained silicon

Stacking faults

Misfit dislocations

\begin{abstract}
A B S T R A C T
The production of low resistance ultra-shallow junctions for e.g. source/drain extensions using low energy ion-implantation will be required for future CMOS devices [H. Wakabayashi, M. Ueki, M. Narihiro, T. Fukai, N. Ikezawa, T. Matsuda, K. Yoshida, K. Takeuchi, Y. Ochiai, T. Mogami, T. Kunio, Trans. Electron Devices 49 (2002) 89-94]. This architecture will require implants which demonstrate high electrical activation and $\mathrm{nm}$ range depth profiles. We investigate the properties of $\mathrm{Sb}$ implants in tensile strained silicon due to their potential to satisfy these criteria and the mobility enhancements associated with tensile strained silicon. Low energy (in this case $2 \mathrm{keV}$ ) implants coupled with Sb's large atomic radius are capable of providing $\sim 10 \mathrm{~nm}$ implant depths. In addition to this, Sb demonstrates higher electrical activation in the presence of tensile strain, when compared with the more traditional n-type dopant As [N.S. Bennett, N.E.B. Cowern, A.J. Smith, R.M. Gwilliam, B.J. Sealy, L. O’Reilly, P.J. McNally, G. Cooke, H. Kheyrandish, Appl. Phys. Lett. 89(2006) 182122]. We now report on the initial results of an ongoing systematic study over a wide silicon tensile strain range (from $0.4 \%$ to $1.25 \%$ strain) in order to establish clear strain-related trends. Graded $\mathrm{Si}_{1-x} \mathrm{Ge}_{x}$ virtual substrates (VS) with $0.1 \leq x \leq 0.3$ were used as template substrates upon which tensile Si layers were grown. Prior to implantation the quality of the strained layer and SiGe buffer is assessed using UV micro-Raman spectroscopy ( $\mu R S$ ), synchrotron X-ray topography (SXRT) and high-resolution X-ray diffraction (HR-XRD). For measurements of strain following implantation, HR-XRD is found to be more useful than $\mu$ RS because of additional carrier-concentration induced Si Raman peak shifts. These shifts obscure small changes in the strain state, and are a result of the degenerate doping levels achieved in these samples $\left(\sim 7 \times 10^{20} \mathrm{~cm}^{-3}\right)$. Using X-ray techniques, at Ge concentrations $>23 \%$ (i.e. $\varepsilon>0.9 \%$ ) we find clear evidence of tilt in the SiGe VS, which impacts on the quality of the strained Si. Additionally, stacking faults have been detected non-destructively in the higher strain samples $\left(\varepsilon=1.25 \%, \mathrm{VS}=\mathrm{Si}_{0.7} \mathrm{Ge}_{0.3}\right)$ using SXRT in transmission mode.
\end{abstract}

(c) 2008 Elsevier B.V. All rights reserved.

\section{Introduction}

The scaling of the CMOS transistor has been the primary driving factor in the performance improvements, and decreasing power consumption, of integrated circuits [3]. The continuation of these improvements will require the production of ultra-shallow junctions for the source/drain extension regions using low energy ion-implantation [1]. Strain engineering using Si/SiGe heterostructures has become a key technology for the enhancement of device operating speeds [4]. Biaxial tensile strain results in the reduction

\footnotetext{
* Corresponding author. Tel.: +353 17005872 .

E-mail address: horan.ken@gmail.com (K. Horan).
}

of the sheet resistance $\left(R_{\mathrm{S}}\right)$ of highly doped n-type layers created by a low energy ( $2 \mathrm{keV})$ As or Sb ion-implantation. The effect is more pronounced for $\mathrm{Sb}$, as $\mathrm{R}_{\mathrm{S}}$ lowering results not only from strainenhanced mobility, but also from an improvement in Sb solubility with strain present [2]. In a comparison between Sb implants in bulk and strained $\mathrm{Si}$, we found that a $0.7 \%$ tensile strain results in a more than doubling of electrically active Sb subject to a low thermal budget rapid thermal anneal (RTA) of $600-800^{\circ} \mathrm{C}$. The fact that these enhancements are independent of scaling alleviates some of the pressures associated with the impending technological and fundamental limitations of device down-sizing [5]. In order for these two methods of performance enhancement to work in concert, it is necessary to gain an understanding of the effects of strain on dopant diffusion and activation at high dopant concentrations. 


\section{Experimental details}

Strained silicon epilayers were grown to thicknesses ranging between $9 \mathrm{~nm}$ and $42 \mathrm{~nm}$. Wafers were implanted with a 2-keV $\mathrm{Sb}$ or As ion dose of between $1 \times 10^{14} \mathrm{~cm}^{-2}$ and $10 \times 10^{14} \mathrm{~cm}^{-2}$ creating a junction at a depth of around $10 \mathrm{~nm}$ in each case. Control samples were prepared using conventional p-type Si wafers for comparison. The implanted dose was measured by Rutherford back-scattering (RBS). Dopant activation was achieved by RTA of the wafer pieces for $10 \mathrm{~s}$ in $\mathrm{N}_{2}$ in the range $600-800^{\circ} \mathrm{C}$.

The quality of the SiGe substrates was investigated using whitebeam synchrotron X-ray topography. The SXRT measurements were performed at the Hamburger Synchrotronstrahlungslabor am Deutschen Elektronen-Synchrotron (HASYLAB-DESY) utilising the continuous spectrum of synchrotron radiation from the DORIS storage ring-bending magnet. The ring operates at positron energies of $4.45 \mathrm{GeV}$ and at typical currents of $80-150 \mathrm{~mA}$. Room temperature micro-Raman measurements were performed with a Jobin Yvon LabRam HR800 system in back-scattering geometry using a 325-nm He-Cd UV laser excitation with a spot size of approximately $1 \mu \mathrm{m}$. High-resolution X-ray diffraction measurements were performed on a Philips X'Pert Pro diffractometer. Reciprocal space maps (RSM) were collected with a three-bounce germanium analyzer crystal placed before the detector, which narrows the acceptance angle to approximately 12 arc s. Peak carrier concentrations were measured by the differential Hall profiling technique [2].

\section{Results and discussion}

\subsection{Micro-Raman spectroscopy}

In performing the $\mu \mathrm{RS}$ measurements additional procedural steps were taken. Before measurement, the silicon Raman peak amplitude was normalised to that of the calibration peak by means of accumulation-time scaling. Post-measurement, the peak positions were adjusted with reference to the $332-\mathrm{nm}$ neon emission line. These additional experimental steps resulted in a reduction of the measurement error by almost an order of magnitude to $\sim 0.1 \mathrm{~cm}^{-1}[6]$.

In a related paper [7] we have shown that the usefulness of micro-Raman spectroscopy as a strain metrology tool becomes limited under conditions of high carrier concentration. This is due to the interaction between the discrete Raman transitions and the continuous free-electron excitations at high carrier concentrations [8-10]. The result of this is a direct dependence of the Si Raman peak shifts on carrier concentration and Fig. 1 gives an example of this.

Fig. 1 shows a plot of the Si Raman peak shift for $0.7 \%$ tensile strained Si samples annealed at $600^{\circ} \mathrm{C}, 700^{\circ} \mathrm{C}$ and $800^{\circ} \mathrm{C}$, relative to their respective as - implanted samples, as a function of carrier concentration. This data shows a clear correlation between these two properties. Due to this dependence, $\mu$ RS is a less effective tool for strain metrology at high carrier concentrations and therefore complementary techniques such as HR-XRD are required for the task under these conditions. Raman spectroscopy however remains a reliable and quick method of measuring material strain in unimplanted samples.

Fig. 2 shows the tensile strain calculated from measurements of the shift in the silicon Raman peak position for samples with Ge substrate content ranging between $10 \%$ and $30 \%$. The strain is calculated using the equation [11,12]

$\sigma_{x x}=\sigma_{y y}=\frac{-\Delta \omega_{\mathrm{SiUV}}}{4}(\mathrm{GPa})$

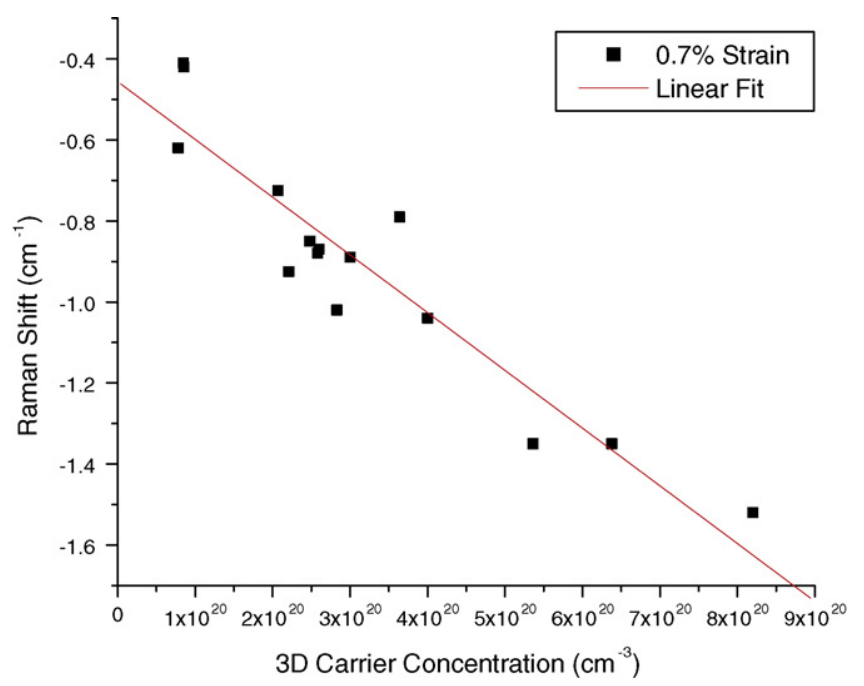

Fig. 1. Post-anneal Raman shift as a function of carrier concentration. Results are shown for strained silicon samples with $0.7 \%$ strain implanted with $\mathrm{Sb}$ doses in the range between $1 \times 10^{14} \mathrm{~cm}^{-1}$ and $1 \times 10^{15} \mathrm{~cm}^{-1}$ and annealed for $10 \mathrm{~s}$ at $600{ }^{\circ} \mathrm{C}$, $700{ }^{\circ} \mathrm{C}$ and $800^{\circ} \mathrm{C}$

where $\sigma_{x x}$ and $\sigma_{y y}$ are the strains in the $x$ and $y$ directions and $\Delta \omega_{\text {SiUv }}$ is the strain induced shift in the Raman peak position. The theoretical strains resulting from the appropriate substrate Ge contents are also plotted, under the assumption of $100 \%$ relaxation in the VS, these are calculated using the equation, [13]

$\sigma_{\mathrm{Si}}=\frac{E_{\mathrm{f}} m}{1-v}(\mathrm{GPa})$

where $E_{\mathrm{f}}$ and $v$ are the Young's modulus and Poisson's ratio of the film, respectively, and $m$ is the lattice misfit between the relaxed virtual substrate and the strained silicon layer. Fig. 2 reveals good agreement between the theory and practice, as all data points but one lie within the experimental error of the theoretical maximum strain. We also observe no appreciable strain relaxation in the super critically thick $\mathrm{Si}_{0.7} \mathrm{Ge}_{0.3}$ samples (tensile strained silicon layer thicknesses of $12 \mathrm{~nm}$ and $15 \mathrm{~nm}$ ). This suggests that at least initially these structures are meta-stable which allows the inclu-

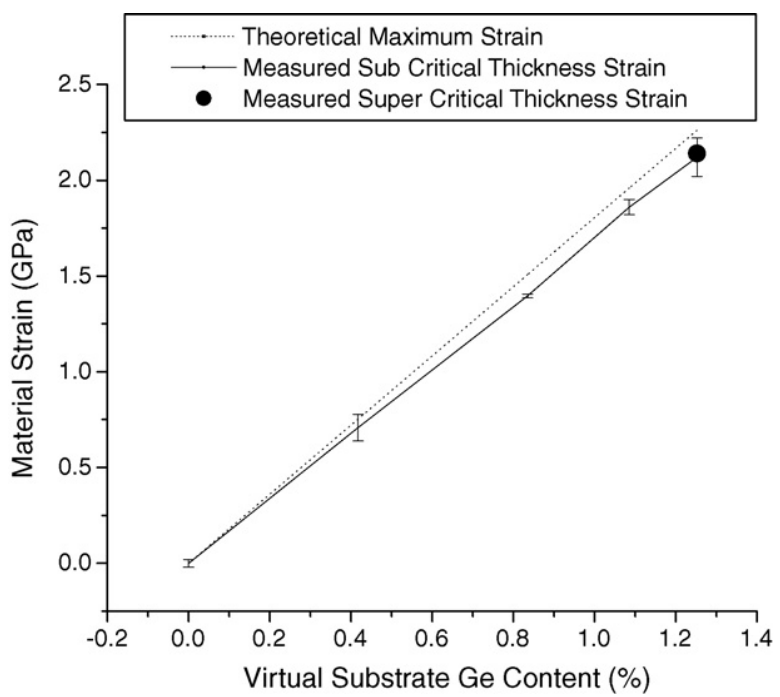

Fig. 2. Measured strain and theoretical maximum strain as a function of Ge substrate content. Lines represent the measured strain and theoretical maximum strain for below critical thickness silicon epilayers, points represent measurements performed on samples with thicknesses above critical thicknesses. 


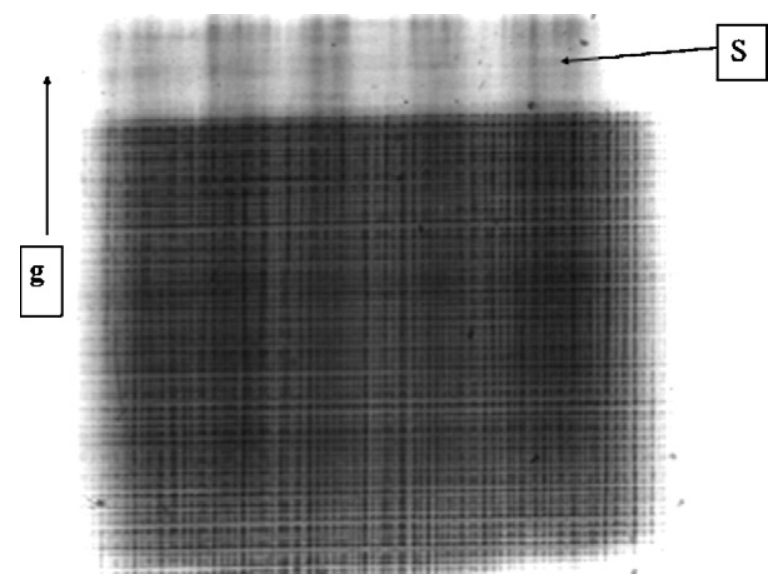

Fig. 3. Large area back reflection topograph of a $\mathrm{Si}_{0.77} \mathrm{Ge}_{0.23}$ substrate and a $12-\mathrm{nm}$ strained silicon epilayer. The reflection shown is the $1 \overline{1} 7$ reflection with a penetration depth of $15.8 \mu \mathrm{m}$. The film-to-sample distance was $80 \mathrm{~mm}$. The topograph was recorded at HASYLAB-DESY synchrotron under white-beam conditions. The projection of the diffraction vector $g$ onto the plane of the recording film is also indicated.

sion of the complete implant profile within the strained silicon epilayer.

\subsection{Synchrotron $X$-ray topography and $H R-X R D$}

By way of example Fig. 3 shows a $1 \overline{1} 7$ large area back reflection topograph of the strained silicon sample grown on the $\mathrm{Si}_{0.77} \mathrm{Ge}_{0.23}$ substrate. The entire SiGe buffer layer $(\sim 3 \mu \mathrm{m})$ is imaged here as the X-ray penetration depth $\left(t_{\mathrm{p}}\right)$ for this $1 \overline{1} 7$ reflection is $15.8 \mu \mathrm{m}$. Two major features of note are observed in the topograph. Firstly, the crosshatched pattern running along two perpendicular $\langle 110\rangle$ directions. This is an image of the array of misfit dislocations (MD) common in strained silicon samples [14]. These MDs provide strain relaxation in the VS, necessary for stable strained silicon epilayer growth. This strain relaxation is further confirmed by reciprocal space map data. These misfit dislocations are observed in all samples. The second feature in the topograph is the presence of a lighter intensity, overlapping diffraction image, labelled " $S$ " in Fig. 3. This displaced image indicates that a region of the SiGe buffer is tilted with respect to the substrate, this tilting results in a different Bragg angle for this region and therefore a slight displacement on the recording film. This tilt is further confirmed by HR-XRD. Fig. 4 shows the 224 RSM in the ( $1 \overline{1} 0)$ diffraction plane. This RSM shows four peaks in the SiGe buffer caused by the terraced graded growth method employed in fabricating these samples and finally the constant composition SiGe peak (most intense) at the maximum $\mathrm{Ge}$ concentration of $23 \%$. The fact that the in-plane reciprocal position of the uppermost of these lies to the right of the silicon substrate peak in the RSM is a result of tilt in the SiGe buffer layer. This tilt can be quantified by examining the 004 omega $(\omega)$ scans in the (110), ( $\left.\begin{array}{lll}1 & 1 & 0\end{array}\right),\left(\begin{array}{lll}1 & 1 & 0\end{array}\right)$ and $\left(\begin{array}{lll}1 & 1 & 0\end{array}\right)$ diffraction planes in accordance with the technique laid out by Nagai [15]. It was found that just above the substrate the SiGe graded layer demonstrates a tilt of $0.16^{\circ}$ toward the $\left[\begin{array}{lll}1 & 1 & 0\end{array}\right]$ lattice plane and that this reduces to $\sim 0.1^{\circ}$ at the start of the strained silicon epilayer. A tilt of $\sim 0.11^{\circ}$ was measured at the uppermost terrace of the SiGe graded layer accounting for its initially anomalous position. An important caveat is that Nagai's theory no longer holds after the formation of MDs; this may account for the reduction in the tilt as the MDs act to not only reduce strain but also lattice tilt. The strained silicon lateral lattice constant can also be seen to be in good agreement with the SiGe buffer in Fig. 4 as their $x$-and $y$-reciprocal space positions can be seen to be the same. Initial HR-XRD data suggests that greater tilt angles are observed

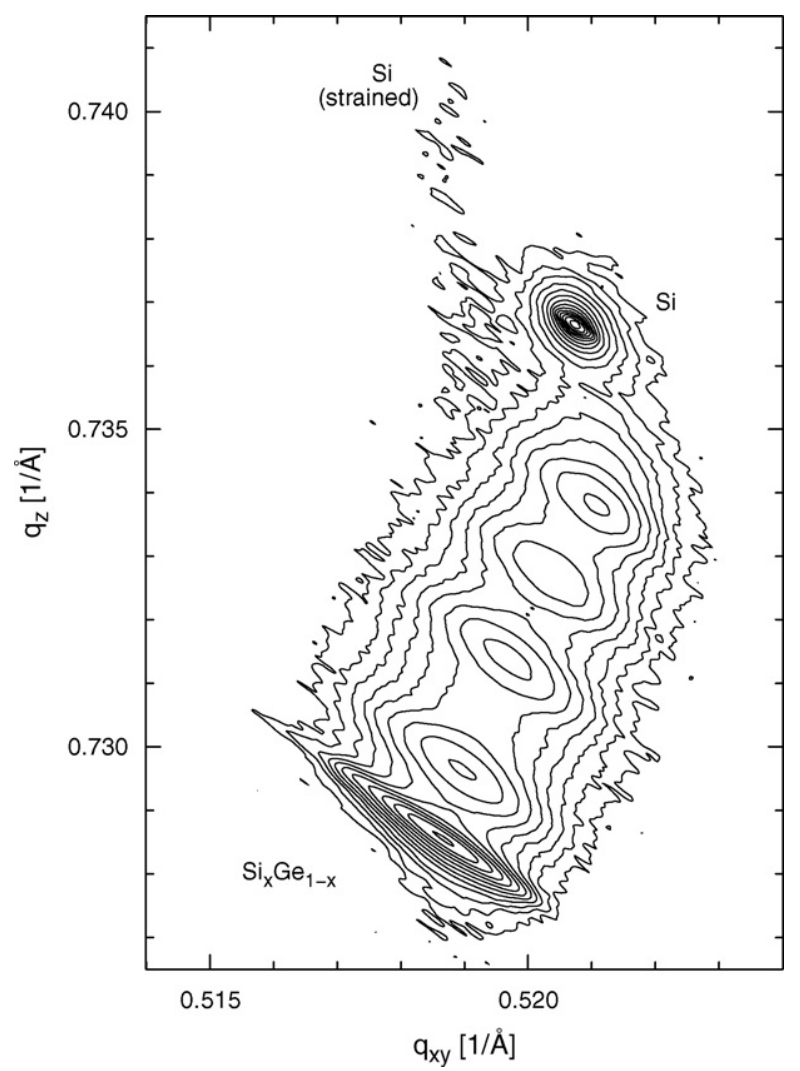

Fig. 4. 224 reciprocal space map (RSM) in the ( 110 ) diffraction plane of the $23 \% \mathrm{Ge}$ virtual substrate sample with $12 \mathrm{~nm}$ strained silicon epilayer. Intensity (a.u.) doubles between contour lines.

for higher Ge concentrations in the VS, reaching a maximum for $\mathrm{Si}_{0.7} \mathrm{Ge}_{0.3}$ substrate, this is in-line with theory as the degree of tilt is dependent on the lattice mismatch [15].

Fig. 5 shows a large area transmission topograph of the strained silicon sample grown on a $30 \%$ Ge virtual substrate. The large "boxlike" structures bounded by misfit dislocations are indicative of stacking faults (SF) [16]. The clarity of these X-ray topographic SF images suggests that the crossing stacking faults are not interfering with each other. We speculate that this may be a result of the graded nature of the SiGe buffer, which can confine MD arrays and SFs to individually grown terraces and this can allow SF to pass under and over each other. The white "boxes" which can be seen in Fig. 5

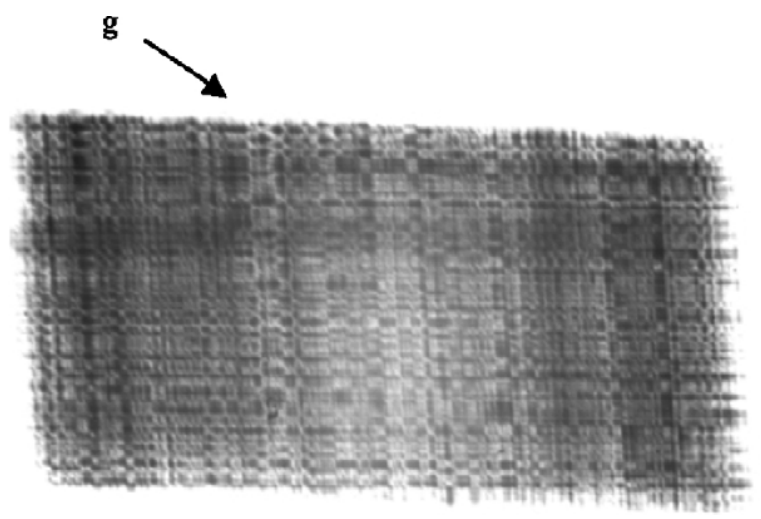

Fig. 5. Large area transmission topograph of the $\mathrm{Si}_{0.3} \mathrm{Ge}_{0.7}$ virtual substrate with a 9-nm strained silicon epilayer. The topograph was recorded at HASYLAB-DESY synchrotron under white-beam conditions with a film-to-sample distance of $80 \mathrm{~mm}$. The projection of the diffraction vector $g$ onto the plane of the recording film is also indicated. 
may be a result of an even number of SF with symmetrically opposite Burgers vectors passing through the imaged region. In this case the two diffracted X-ray wave-fronts could undergo phase-shifts in opposite directions resulting in no net change. For example, four stacking faults on top of each other, bordered by $60^{\circ}$ misfit dislocations having the same Burgers vectors, may produce a contrast similar to that imaged in Fig. 5.

\section{Conclusion}

$\mu R S$, SXRT and HR-XRD were used to study the stability of highly strained tensile silicon layers doped with $\mathrm{Sb}$, grown on $\mathrm{Si}_{1-x} \mathrm{Ge}_{x}$ virtual substrates. For unimplanted samples $\mu$ RS continues to be of major benefit for strain metrology. The Raman data collected here shows the strain present in these samples to be close to the theoretical maximum strain for these structures. We also see that at least initially, structures grown to thicknesses beyond their theoretical critical thickness are meta-stable. SXRT topographs reveal the presence of misfit dislocations in all samples confirming the presence of a relaxed VS for all Ge concentrations. The emergence of lattice tilts at VS Ge contents above $\sim 23 \%$ have been detected in both SXRT and HR-XRD measurements, and this may be of technological concern as this tilt continues into the strained silicon layer. At a VS Ge content of 30\% stacking faults have been imaged using SXRT. These appear to be confined by the misfit dislocations in the SiGe VS. However, if these were to reach the strained silicon layer they could cause major problems for any device structure built on this material and future studies will focus on their thermal stability.

\section{Acknowledgements}

Science Foundation Ireland is gratefully acknowledged for funding this project under the Investigator Programme Grant Scheme
Project 05/IN/I656. We acknowledge HASYLAB and the European Community for funding under Contract RII3-CT-2004_506009 (IASFS). We are grateful to T. Wroblewski and C. Paulmann for their help at HASYLAB beamline F-1.

\section{References}

[1] H. Wakabayashi, M. Ueki, M. Narihiro, T. Fukai, N. Ikezawa, T. Matsuda, K. Yoshida, K. Takeuchi, Y. Ochiai, T. Mogami, T. Kunio, Trans. Electron Devices 49 (2002) 89-94.

[2] N.S. Bennett, N.E.B. Cowern, A.J. Smith, R.M. Gwilliam, B.J. Sealy, L. O'Reilly, P.J McNally, G. Cooke, H. Kheyrandish, Appl. Phys. Lett. 89 (2006) 182122.

[3] M. Ieong, B. Doris, J. Kedzierski, K. Rim, M. Yang, Science 306 (2004) $2057-$ 2060.

[4] M.L. Lee, E.A. Fitzgerald, M.T. Bulsara, M.T. Currie, A. Lochtefeld, J. Appl. Phys. 97 (2005) 011101.

[5] H. Iwai, Solid-State Circuits IEEE J. Solid-State Circuits 34 (1999) 357-366.

[6] K. Horan, L. O’Reilly, N.S. Bennett, P.J. McNally, B.J. Sealy, N.E.B. Cowern, Optimization of UV-Raman spectroscopy analysis of ultra-shallow junctions in strained silicon, in: IOM3 3rd International Conference on Nanomaterials and Nanomanufacturing, 2007.

[7] L. O’Reilly, K. Horan, P.J. McNally, N.S. Bennett, N.E.B. Cowern, A. Lankinen, B.J. Sealy, R.W. Gwillian, Appl. Phys. Lett. 92 (2008) 233506.

[8] F. Cerdeira, M. Cardona, Phys. Rev. B 5 (1972) 1440.

[9] M. Chandrasekhar, M. Cardona, E.O. Kane, Phys. Rev. B 16 (1977) 3579.

[10] M. Chandrasekhar, J.B. Renucci, M. Cardona, Phys. Rev. B 17 (1978) 1623.

[11] L. O'Reilly, N.S. Bennett, P.J. McNally, B.J. Sealy, N.E.B Cowern, MicroRaman study of anomalous dopant-induced behavior in ultra-shallow As and $\mathrm{Sb}$ doped strained $\mathrm{Si}$, in: International Workshop on INSIGHT in Semiconductor Device Fabrication, Metrology and Modeling (INSIGHT-2007), 2007.

[12] I. De Wolf, Semicond. Sci. Technol. 11 (1996) 139-154.

[13] T.S. Perova, K. Lyutovich, E. Kasper, A. Waldron, M. Oehme, R.A. Moore, Mater Sci. Eng. B 135 (2006) 192-194.

[14] R. Koehler, H. Raidt, W. Neumann, J.U. Pfeiffer, J. Phys. D: Appl. Phys. 38 (2005) 319-327.

[15] H. Nagai, J. Appl. Phys. 45 (1974) 3789-3794.

[16] A. Authier, Dynamical Theory of X-Ray Diffraction, Oxford University Press Inc. New York, 2001 